\title{
Mitigation of the Hose Instability in Plasma-Wakefield Accelerators
}

\author{
T. J. Mehrling, ${ }^{1,2, *}$ R. A. Fonseca, ${ }^{2,3}$ A. Martinez de la Ossa, ${ }^{1}$ and J. Vieira ${ }^{2,}$ \\ ${ }^{1}$ Institut für Experimentalphysik, Universität Hamburg, 22761 Hamburg, Germany \\ ${ }^{2}$ GoLP/Instituto de Plasmas e Fusão Nuclear, Instituto Superior Técnico, Universidade de Lisboa, 1049-001 Lisboa, Portugal \\ ${ }^{3}$ DCTI/ISCTE Instituto Universitário de Lisboa, 1649-026 Lisbon, Portugal
}

(Received 12 July 2016; revised manuscript received 31 January 2017; published 26 April 2017)

\begin{abstract}
Current models predict the hose instability to crucially limit the applicability of plasma-wakefield accelerators. By developing an analytical model which incorporates the evolution of the hose instability over long propagation distances, this work demonstrates that the inherent drive-beam energy loss, along with an initial beam-energy spread, detunes the betatron oscillations of beam electrons and thereby mitigates the instability. It is also shown that tapered plasma profiles can strongly reduce initial hosing seeds. Hence, we demonstrate that the propagation of a drive beam can be stabilized over long propagation distances, paving the way for the acceleration of high-quality electron beams in plasma-wakefield accelerators. We find excellent agreement between our models and particle-in-cell simulations.
\end{abstract}

DOI: 10.1103/PhysRevLett.118.174801

Plasma-based accelerators can provide accelerating fields in excess of $10 \mathrm{GV} / \mathrm{m}[1,2]$. As a result, these devices can potentially contribute to a future generation of more compact particle accelerators and radiation sources. Plasma-wakefield accelerators (PWFAs) [3,4] employ charged particle beams as drivers of large-amplitude plasma waves. Significant experimental results $[2,5]$ were obtained in the blowout regime, in which a particle beam with a charge density greater than the ambient plasma density expels all plasma electrons within its vicinity, thereby generating a copropagating ion channel with linear electron focusing and extreme accelerating fields [6].

Identified by Whittum et al. in the early 1990s [7], the hose instability (HI) remains a long-standing challenge for PWFAs. Hosing is seeded by initial transverse asymmetries of the beam or plasma phase-space distributions. According to current models, the beam-centroid displacement is amplified exponentially during propagation in the plasma [7-11], ultimately leading to a beam breakup. The most recent description for the coupled evolution of the ionchannel centroid $X_{c}(\xi, t)$ and the beam centroid $X_{b}(\xi, t)$ in the blowout regime is given by [11]

$$
\begin{gathered}
\frac{\partial^{2} X_{c}}{\partial \xi^{2}}+\frac{k_{p}^{2} c_{\psi}(\xi) c_{r}(\xi)}{2}\left(X_{c}-X_{b}\right)=0 \\
\frac{\partial^{2} X_{b}}{\partial t^{2}}+\omega_{\beta}^{2}\left(X_{b}-X_{c}\right)=0
\end{gathered}
$$

with the time $t$ and the comoving coordinate $\xi=c t-z$, where $z$ is the longitudinal coordinate and $c$ is the speed of light. The plasma wave number is denoted by $k_{p}=\omega_{p} / c$, and the betatron frequency by $\omega_{\beta}=\omega_{p} / \sqrt{2 \gamma}$, with the Lorentz factor $\gamma$, where $\omega_{p}=\sqrt{4 \pi n_{0} e^{2} / m}$ is the plasma frequency with the ambient plasma density $n_{0}$, the elementary charge $e$, and the electron rest mass $m$. The coefficients $c_{\psi}(\xi)$ and $c_{r}(\xi)$ account for the relativistic motion of electrons in the blowout sheath and for a $\xi$ dependence of the blowout radius and the beam current [11]. According to Eq. (1), a beam-centroid displacement $X_{b}$ leads to a displacement of the ion-channel centroid $X_{c}$ along the beam. The displacement $X_{c}$ couples back to the temporal evolution of $X_{b}$ according to Eq. (2). The case where $c_{\psi}=c_{r}=1$ recovers the seminal hosing model [7]. This limit, which accounts for a linear response of sheath electrons, is characterized by an exponential growth of $X_{b}$ and $X_{c}$ with increasing $\xi$ and $t[8,9]$. Owed to the nonlinear response of the electron sheath, the growth rates decrease in the blowout regime, because $c_{\psi} c_{r}<1$ [11]. Despite this reduction of the growth rate, however, the current theoretical descriptions still predict that hosing eventually results in a beam breakup and, hence, poses a strong constraint for the applicability of PWFAs.

Although in agreement with particle-in-cell (PIC) simulations for short propagation distances [11], current models overestimate the hosing growth rates once the drive-beam energy change becomes significant. This is shown in Fig. 1, which depicts the result of a threedimensional (3D) PIC simulation with OSIRIS [12], indicating that hosing can be far less pronounced than what has been reported so far. Yet unnoticed, this intriguing result suggests that the blowout regime can provide a saturation mechanism for the HI, which strongly damps the beamcentroid oscillations during the propagation, thereby contributing to the stabilization of the beam propagation over long distances.

In this Letter, we show by means of an analytical theory and with PIC simulations that hosing can be mitigated in the blowout regime. This has not previously been identified, because current analytic models neglect the energy 


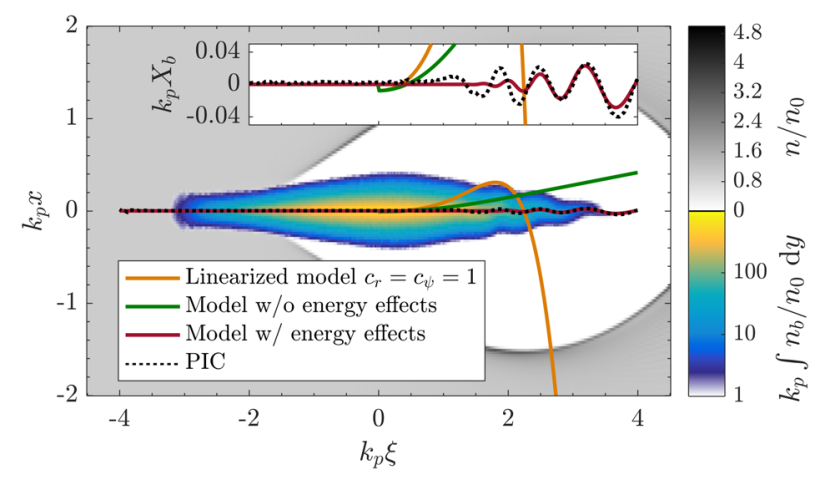

FIG. 1. Result from a 3D PIC simulation showing plasma and beam charge densities at the time $\omega_{\beta, 0} t=71.6$. The beam has an initial spatial centroid offset, introduced at the position $\xi=0$, and is subject to hosing. Beam charge density $n_{b}$ is projected onto the shown $x-\xi$ plane. Lines indicate $X_{b}(\xi)$, as a result from the models in Refs. [7,8] (orange solid curve) and [11] (green solid curve), respectively. Depicted is also the result from Eqs. (1) and (7), derived within this work (red solid curve), and $X_{b}(\xi)$ retrieved from the PIC simulation (black dashed curve). Inset: Enlarged depiction of the beam centroids.

change of the drive-beam particles. Instead, here we find that the energy change, which naturally occurs as the beam excites the plasma wave, and/or an initial beam-energy chirp, can detune the betatron oscillations of individual slices along the beam, thereby mitigating their resonant coupling via the plasma. We also show that beam-centroid oscillations can significantly be reduced if the drive beam features a subpercent uncorrelated energy spread, which introduces a decoherence of the betatron oscillations of individual beam electrons. Our theoretical model can accurately explain the reduced centroid amplitude of oscillations observed in the simulations, as shown in Fig. 1 (see the dashed line and solid red line) and in Fig. 2. We also propose to substantially decrease the initial hosing seed by using tailored vacuum-to-plasma transitions. We confirm all our analytical predictions with 3D PIC simulations using OSIRIS. The parameters used in these simulations differ from those proposed for a number of high-energy beam facilities. Numerical demonstrations of the hosing saturation for parameters corresponding to these facilities are to be published elsewhere [13]. Our findings pave the way for the stable acceleration of high-quality beams over long distances in PWFAs and provide theoretical evidence for why hosing to date has not been experimentally detected.

The starting point is the differential equation for the transverse position $x$ of a single beam electron relative to the axis in a homogeneous ion channel $[14,15]$ :

$$
\frac{d^{2} x}{d t^{2}}+\frac{\dot{\gamma}}{\gamma} \frac{d x}{d t}+\omega_{\beta}^{2}\left(x-X_{c}\right)=0,
$$

where $\dot{\gamma}=d \gamma / d t$. The Lorentz factor $\gamma \simeq p_{z} / m c \gg 1\left(p_{z}\right.$ refers to the longitudinal momentum) is decoupled from the transverse motion, since $d x / d t \ll c$. The term $\dot{\gamma} / \gamma$ results in a damping or amplification of the amplitude of the single-electron oscillation, depending on whether the electron gains $(\dot{\gamma}>0)$ or loses $(\dot{\gamma}<0)$ energy, respectively. The restoring force is directed towards the channel centroid $X_{c}$. The solution for Eq. (3) is

$$
\begin{aligned}
x(t) \simeq & x_{0} A(t) \cos [\varphi(t)]+\frac{p_{x, 0}}{m \gamma_{0} \omega_{\beta, 0}} A(t) \sin [\varphi(t)] \\
& +\omega_{\beta, 0} \int_{0}^{t} A(t) A\left(t^{\prime}\right) \sin \left[\varphi(t)-\varphi\left(t^{\prime}\right)\right] X_{c}\left(t^{\prime}\right) d t^{\prime},
\end{aligned}
$$

where $\omega_{\beta, 0}=\omega_{p} / \sqrt{2 \gamma_{0}}, A(t)=\left[\gamma_{0} / \gamma(t)\right]^{1 / 4}$, and $\gamma_{0}$ and $p_{x, 0}$ are the initial Lorentz factor and transverse momentum, respectively. The phase advance is defined by $\varphi(t)=\int \omega_{\beta} d t$. The relative energy and amplitude variations occur on time scales longer than the betatron period in relevant scenarios. Thus, the terms $\left|\dot{\gamma} \dot{A} /\left(\dot{\varphi}^{2} \gamma A\right)\right| \ll 1$, $\left|\ddot{A} /\left(\dot{\varphi}^{2} A\right)\right| \ll 1$, and $\left|\dot{\gamma} / 4 \gamma_{0} \omega_{\beta, 0}\right| \ll 1$ were neglected.

In the following, the energy of an electron is given by $\gamma(t)=\overline{\gamma_{0}}+\mathcal{E} t+\delta \gamma$, where $\overline{\gamma_{0}}=\overline{\gamma_{0}}(\xi)$ is the initial mean slice energy as a function of the comoving coordinate, accounting for an initial energy chirp. The differential change of energy along the beam is accounted by the term $\mathcal{E} t$, where $\mathcal{E}=-e E_{z} / m c$, where $E_{z}=E_{z}(\xi)$ is the longitudinal electric field and where electrons are fixed to their initial position in the comoving frame. The uncorrelated energy spread is incorporated through a finite deviation of the electron energy from the mean slice energy $\delta \gamma=\gamma-\bar{\gamma}$. All overlined quantities refer to slice-averaged quantities.

Electrons with a small relative energy deviation $|\delta \gamma / \bar{\gamma}| \ll 1$ have a betatron frequency $\omega_{\beta}$ which deviates from $\overline{\omega_{\beta}}$ according to $\omega_{\beta} \simeq \overline{\omega_{\beta}}(1-\delta \gamma / 2 \bar{\gamma})$. Hence,

$$
\varphi(t)=\bar{\varphi}(t)\left(1-\frac{\delta \gamma}{2 \overline{\gamma_{0}}} \frac{\overline{\omega_{\beta}}}{\overline{\omega_{\beta, 0}}}\right)
$$

where $\quad \bar{\varphi}=2\left(\overline{\omega_{\beta, 0}} / \overline{\omega_{\beta}}-1\right) / \epsilon, \quad \overline{\omega_{\beta, 0}}=\omega_{p} / \sqrt{2 \overline{\gamma_{0}}}, \quad$ and $\overline{\omega_{\beta}}=\overline{\omega_{\beta, 0}} / \sqrt{1+\epsilon \overline{\omega_{\beta, 0}} t}$. Note that $\overline{\omega_{\beta}}$ is time dependent owing to a finite relative energy change per betatron cycle $\epsilon=\mathcal{E} / \overline{\gamma_{0}} \overline{\omega_{\beta, 0}}=-\sqrt{2 / \overline{\gamma_{0}}} E_{z} / E_{0}$, with $E_{0}=\omega_{p} m c / e$. Equation (5) infers that electrons with differing energy within a slice acquire a differing phase advance, which leads to the phase mixing of the betatron oscillations along the beam. This phase mixing can damp the HI, similarly to the HI damping occurring in fully self-modulated beams through a change of the betatron frequency for each self-modulated beamlet [16].

To assess the effect of the phase mixing onto the HI, the beam centroid $X_{b}$ is deduced from Eq. (4) by averaging with respect to an initial phase-space distribution $f_{0}\left(x_{0}, p_{x, 0}, \gamma_{0}\right)$ within each beam slice, $X_{b}(\xi, t)=\int x f_{0} d x_{0} d p_{x, 0} d \gamma_{0}$, with $\int f_{0} d x_{0} d p_{x, 0} d \gamma_{0}=1$. We assume that the initial transverse 
offset and momentum in a slice are not correlated with energy. Hence, $f_{0}$ is separable: $f_{0}=f_{\perp}\left(x_{0}, p_{x, 0}\right) f_{\gamma}\left(\gamma_{0}\right)$. While the distribution $f_{\perp}\left(x_{0}, p_{x, 0}\right)$ is arbitrary (apart from assuming $f_{\perp}=0$ outside the channel) with a mean spatial value $\overline{x_{0}}=X_{b, 0}$, the energy distribution considered is Gaussian and given by $f_{\gamma}=\left(\sqrt{2 \pi} \sigma_{\gamma}\right)^{-1} \exp \left(-\delta \gamma^{2} / 2 \sigma_{\gamma}^{2}\right)$. Averaging over the initial transverse phase-space distribution and over the Gaussian energy distribution, neglecting the variation of $A$ owed to $\delta \gamma$, yields

$$
\begin{aligned}
X_{b}(\xi, t) \simeq & X_{b, 0}(\xi) \bar{A}(\xi, t) \exp \left(-\frac{\Delta \gamma^{2} \bar{\alpha}(\xi, t)^{2}}{2}\right) \cos [\bar{\varphi}(\xi, t)] \\
& +\int_{0}^{t} \bar{A}(\xi, t) \bar{A}\left(\xi, t^{\prime}\right) \exp \left(-\frac{\Delta \gamma^{2}\left[\bar{\alpha}(\xi, t)^{2}-\bar{\alpha}\left(\xi, t^{\prime}\right)^{2}\right]}{2}\right) \\
& \times \sin \left[\bar{\varphi}(\xi, t)-\bar{\varphi}\left(\xi, t^{\prime}\right)\right] X_{c}\left(\xi, t^{\prime}\right) \overline{\omega_{\beta, 0}}(\xi) d t^{\prime}
\end{aligned}
$$

with the initial relative energy spread $\Delta \gamma=\sigma_{\gamma} / \overline{\gamma_{0}}$, the amplitude $\bar{A}=\left(\overline{\gamma_{0}} / \bar{\gamma}\right)^{1 / 4}$, and $\bar{\alpha}=\bar{\varphi} \overline{\omega_{\beta}} / 2 \overline{\omega_{\beta, 0}}$. The initial mean slice transverse momentum is assumed zero for compactness. Equations (1) and (6) describe the coupled evolution of $X_{c}$ and $X_{b}$ in the blowout regime. They recover known results in the blowout regime at sufficiently early times [11]. When energy effects become relevant, however, they show that hosing can be mitigated.

To investigate the predictions of Eqs. (1) and (6) analytically, we use a two-particle (two-slice) model such that $X_{b}(\xi, t)=X_{b, 1}(\xi, t) \delta\left(\xi-\xi_{1}\right)+X_{b, 2}(\xi, t) \delta\left(\xi-\xi_{2}\right)$. The first slice, at $\xi_{1}$, is unaffected by the HI but drives the channel centroid oscillations according to Eq. (1). The motion of the slice at $\xi_{2}$ is driven by those channel oscillations according to Eq. (6).

We start by determining the time scale for the hosing mitigation by isolating the contributions of finite $\partial_{\xi} \epsilon \neq 0$, which accounts for the differential energy change along the beam. Analytical results are valid for arbitrary $c_{r}(\xi), c_{\psi}(\xi)$, for beams without an initial energy spread, and for constant $\bar{A}$. Initially, the trailing slice is resonantly driven by the transverse motion of the first slice, enhancing the amplitude of $X_{b}\left(\xi_{2}\right)$. This corresponds to the initial hosing growth investigated in Ref. [11]. However, at the time $\bar{\omega}_{\beta, 0} t_{d, \epsilon} \simeq$ $\sqrt{3 \pi / \Delta \epsilon}$, where $\Delta \epsilon=\left|\epsilon\left(\xi_{1}\right)-\epsilon\left(\xi_{2}\right)\right|$, the phase difference of the two slices is significant, and $X_{b}\left(\xi_{2}\right)$ reaches a maximum [13]. For $t>t_{d, \epsilon}$, the oscillation amplitude of $X_{b}\left(\xi_{2}\right)$ saturates at a smaller value. This fundamentally novel result is in strong contrast with current models, which predict exponentially growing amplitudes until beam breakup occurs.

This finding is significant, because the pump depletion time is typically much longer than $t_{d, \epsilon}$. We demonstrate this by comparing the pump depletion time, given by $t_{\mathrm{dp}}=1 / \overline{\omega_{\beta, 0}} \hat{\epsilon}$, to $t_{d, \epsilon}$, where $\hat{\epsilon}=\max (-\epsilon)$. Hence, decoupling of two slices occurs well before pump depletion if $\Delta \epsilon / \hat{\epsilon}>3 \pi \hat{\epsilon}$. Because $|\hat{\epsilon}| \ll 1$ and since $\Delta \epsilon / \hat{\epsilon}$ ranges from zero to unity along any drive beam, the two-particle model suggests that slices within the beam in PWFAs are decoupled significantly before depletion.

The parameter $\epsilon$ is related to key experimental PWFA parameters as follows. The longitudinal field within the beam region is $E_{z} / E_{0} \simeq \sqrt{I_{b} / I_{A}}[17,18]$, where $I_{b}$ is the beam current and $I_{A} \simeq 17 \mathrm{kA}$ is the Alfvén current. Hence, $\epsilon \simeq-\sqrt{2 I_{b} /\left(I_{A} \overline{\gamma_{0}}\right)}$, and Facility for Advanced Accelerator Experimental Tests (FACET) experimental parameters $[19,20]$, for instance, yield $\hat{\epsilon} \approx 0.007$. This indicates that hosing stops well before energy depletion in typical PWFA scenarios and possibly justifies why hosing was not detected in previous experiments [2,5].

The two-particle model also indicates that an initial linear energy chirp $\chi=\gamma_{b}^{-1} k_{p}^{-1} d \bar{\gamma} / d \xi$ can mitigate hosing. The centroid oscillations of two spatially resonant beam slices $\left(\Delta \xi=k_{p}^{-1} \pi \sqrt{2}\right)$ decouple after $\omega_{\beta, b} t_{d, \chi} \simeq \sqrt{2} /|\chi|$, assuming $c_{r} c_{\psi}=1$ and $\epsilon=0$, thus causing HI damping. This is similar to the method of Balakin-NovokhatskySmirnov damping [21]. Here, $\gamma_{b}$ and $\omega_{\beta, b}$ refer to the initial beam-averaged Lorentz factor and betatron frequency, respectively.

Additionally, according to Eq. (6), the $X_{b}$ oscillations are damped exponentially owing to a finite uncorrelated energy spread. To isolate this effect, we consider a beam with no initial chirp in the limit of no slice energy change $(\epsilon \rightarrow 0)$. In this conservative scenario, the amplitude of the centroid oscillations reduces by $\exp (-1 / 2)$ after the decoherence time $\overline{\omega_{\beta, 0}} t_{d, \Delta \gamma} \simeq 2 / \Delta \gamma$ [13]. Therefore, $t_{d, \Delta \gamma} \lesssim$ $t_{\mathrm{dp}}$ if $\Delta \gamma \gtrsim 2 \hat{\epsilon}$. For the typical parameters of FACET, where $\hat{\epsilon} \approx 0.007$, a subpercent-level energy spread already significantly contributes to the mitigation of hosing. It should be noted that, if $t_{d, \epsilon} \lesssim t_{d, \Delta \gamma}$, the exponential damping of $X_{b}$ due to the uncorrelated energy spread becomes substantial, since $X_{b}$ stops growing owing to finite $\partial_{\xi} \epsilon \neq 0$.

Because fully analytical solutions of our model are complex, we complement the above analysis with PIC simulations using OSIRIS [12] and with numerical solutions of Eq. (1) and the differential form of Eq. (6):

$$
\begin{aligned}
& \frac{\partial^{2} X_{b}}{\partial t^{2}}+\frac{{\overline{\omega_{\beta}}}^{2}}{\overline{\omega_{\beta, 0}}}\left(\epsilon+\kappa_{1} \Delta \gamma^{2}\right) \frac{\partial X_{b}}{\partial t} \\
& \quad+{\overline{\omega_{\beta}}}^{2}\left(1+\kappa_{2} \Delta \gamma^{2}\right)\left(X_{b}-X_{c}\right)=0,
\end{aligned}
$$

with $\kappa_{1}=\left(\overline{\omega_{\beta}} / \overline{\omega_{\beta, 0}}-\left(\overline{\omega_{\beta}} / \overline{\omega_{\beta, 0}}\right)^{2}\right) / \epsilon$ and $\kappa_{2}=\left(\overline{\omega_{\beta}} / \overline{\omega_{\beta, 0}}\right)^{4} /$ $2-\left(\overline{\omega_{\beta}} / \overline{\omega_{\beta, 0}}\right)^{3} / 4$. Equation (7), which neglects terms $\mathcal{O}\left(\Delta \gamma^{4}\right)$ and $\mathcal{O}\left(\epsilon^{2}\right)$, applies for any beam in a blowoutregime wakefield.

We consider the example of a Gaussian electron beam with a steplike initial centroid displacement at $\xi=0$, driving a plasma wave in the blowout regime in a homogeneous plasma and being subject to the HI (cf. Fig. 1) [22]. Numerical solutions of Eqs. (1) and (7) 


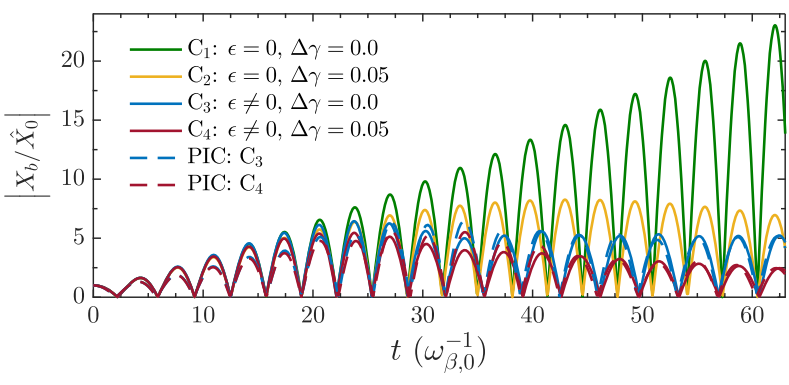

FIG. 2. Absolute value of the beam centroid at $k_{p} \xi=3.0$. Depicted are numerical solutions of Eq. (7) for no energy change $\epsilon=0$ and no energy spread $\Delta \gamma=0.0$ (green solid curve), for $\epsilon=0$ and $\Delta \gamma=0.05$ (yellow solid curve), for $\epsilon \neq 0$ and $\Delta \gamma=$ 0.0 (blue solid curve), and for $\epsilon \neq 0$ and $\Delta \gamma=0.05$ (red solid curve). These curves are compared to the results of PIC simulations (dashed curves).

for this example are depicted in Fig. 2 for the cases $C_{1}$ : $\epsilon=0, \quad \Delta \gamma=0.0 ; \quad C_{2}: \quad \epsilon=0, \quad \Delta \gamma=0.05 ; \quad C_{3}: \quad \epsilon \neq 0$, $\Delta \gamma=0.0$; and $C_{4}: \epsilon \neq 0, \Delta \gamma=0.05$, together with results from PIC simulations for the two latter cases $\left(C_{4}\right.$ also corresponds to the result in Fig. 1). Case $C_{1}$, which resembles the model in Ref. [11], features the expected exponential growth rate, as illustrated in Fig. 2. For $C_{3}$, the detuning of the slice betatron oscillations leads to a saturation of the HI. According to the two-particle model, the maximum amplitude for $C_{3}$ is expected near $\bar{\omega}_{\beta, 0} t_{d, \epsilon} \approx$ 22.7 ( $\Delta \epsilon$ between $k_{p} \xi=0$ and the depicted slice at $k_{p} \xi=3.0$ ), which is in good agreement with the numerical result and the PIC result. Moreover, in $C_{2}$ and $C_{4}$, the centroid oscillations are damped because of the energyspread-induced betatron decoherence within the slices. In $C_{2}$ and $C_{4}$, the energy spread is $\Delta \gamma=0.05$, thus yielding $\overline{\omega_{\beta, 0}} t_{d, \Delta \gamma}=40$. The corresponding exponential damping of $X_{b}$ for $t \gtrsim t_{d, \Delta \gamma}$ is in good agreement with the observations in Fig. 2 for both the numerical solutions of Eqs. (1) and (7) and the PIC simulations.

Effective damping of the HI can occur as long as the hosing seed is sufficiently small not to lead to a beam breakup before the mitigation takes place. Reducing the initial hose seed is therefore still crucial to fully stabilize the driver propagation. For this purpose, we propose a novel concept which employs plasma density tapers to mitigate initial beam-centroid offsets that seed hosing.

We consider a taper of the plasma density from the vacuum-to-plasma interface at position $z_{v}$ to the flattop plasma profile from position $z_{0}$. The beam centroid during the propagation in the tailored vacuum-to-plasma transition is described by

$$
\frac{d^{2} X_{b}}{d z^{2}}+k_{\beta}(z)^{2} X_{b}=0
$$

with $k_{\beta}=k_{\beta, 0} \sqrt{n / n_{0}}$, when neglecting the channel centroid displacement, the beam-energy change, and effects

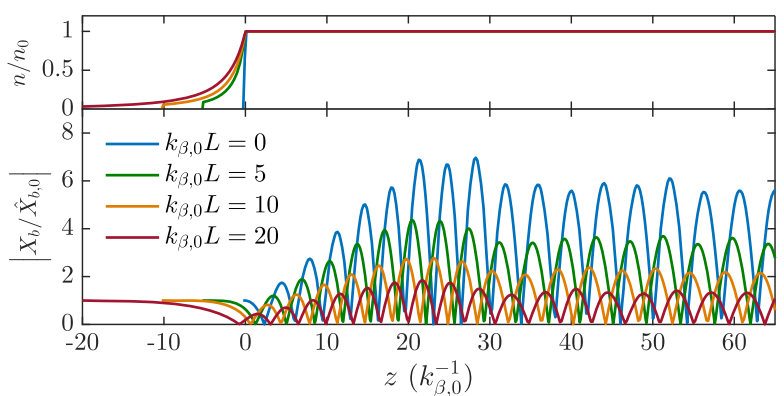

FIG. 3. Hose mitigation by means of plasma density tapers at the tail of a beam $k_{p} \xi=4.0$. Shown are density profiles for different taper lengths (top) and respective beam-centroid amplitudes from PIC simulations (bottom) for $k_{\beta, 0} L=0$ (blue curve), $k_{\beta, 0} L=5$ (green curve), $k_{\beta, 0} L=10$ (orange curve), and $k_{\beta, 0} L=20$ (red curve).

from the energy spread. This equation corresponds to the nonconservative system of an harmonic oscillator with a time-dependent frequency. The beam centroid is therefore damped during the propagation through the taper.

To confirm the hosing seed mitigation scheme, we reran PIC simulations of $C_{3}$ with a tapered plasma density profile. The considered propagation-distance-dependent betatron wave number $k_{\beta}=\omega_{\beta} / c$ is given by $k_{\beta}(z)=$ $k_{\beta, 0}\left[1-(z-z 0) / \lambda_{\text {opt }}\right]^{-2}$ for $z_{v}<z \leq z_{0}, k_{\beta}(z)=k_{\beta, 0}$ for $z>z_{0}$, and $k_{\beta}(z)=0$ otherwise (this functional dependence was used for the beam betatron function matching in Refs. [27,28]). Here $\lambda_{\text {opt }} \simeq L / \sqrt{k_{\beta, 0} L}$ is an optimized characteristic scale length of the taper. Such density profiles can be experimentally realized in appropriate gas capillaries [29]. Figure 3 depicts $X_{b}$ for various taper lengths, illustrating the substantial reduction of hosing when $k_{\beta, 0} L \gtrsim 1$, compared to the case with no taper.

This work demonstrates that the self-consistent beamenergy evolution in the blowout regime can mitigate the hose instability in PWFAs. We show that the drive-beam energy chirp, either introduced prior to the interaction or developed during propagation, results in the mitigation of the HI before the drive beam is energy depleted, regardless of the initial beam energy [13]. We also find that a subpercent uncorrelated energy spread will further reduce the centroid oscillations. Furthermore, it is shown that tapering the plasma profile can efficiently reduce the initial hose seed.

We acknowledge the grant of computing time by the Jülich Supercomputing Centre on JUQUEEN under Project No. HHH23 and the use of the DESY IT high-performance computing facilities. This work was supported by LaserLab Europe IV Grant Agreement No. 654148 (H2020INFRAIA-2014-2015) and EuPRAXIA Grant Agreement No. 653782 (H2020-INFRADEV-1-2014-1). The work leading to this publication was supported by the German Academic Exchange Service (DAAD) with funds from the 
German Federal Ministry of Education and Research (BMBF) and the People Program (Marie Curie Actions) of the European Union's Seventh Framework Program (FP7/2007-2013) under REA Grant Agreement No. 605728 (P.R.I.M.E., Postdoctoral Researchers International Mobility Experience). J. V. acknowledges the support of FCT (Portugal) Grant No. SFRH/IF/01635/2015.

*timon.mehrling@desy.de jorge.vieira@ist.utl.pt

[1] A. Modena, Z. Najmudin, A. Dangor, C. Clayton, K. A. Marsh, C. Joshi, V. Malka, C. Darrow, C. Danson, and F. Neely, and D. Walsh, Nature (London) 377, 606 (1995).

[2] I. Blumenfeld, C. E. Clayton, F.-J. Decker, M. J. Hogan, C. Huang, R. Ischebeck, R. Iverson, C. Joshi, T. Katsouleas, N. Kirby, W. Lu, K. A. Marsh, W. B. Mori, P. Muggli, E. Oz, R. H. Siemann, D. Walz, and M. Zhou, Nature (London) 445, 741 (2007).

[3] V. I. Veksler, in Proceedings of the CERN Symposium on High-Energy Accelerators, Pion Physics, 1956 (unpublished), p. 80.

[4] P. Chen, J. M. Dawson, R. W. Huff, and T. Katsouleas, Phys. Rev. Lett. 54, 693 (1985).

[5] M. Litos et al., Nature (London) 515, 92 (2014).

[6] J. B. Rosenzweig, B. Breizman, T. Katsouleas, and J. J. Su, Phys. Rev. A 44, R6189 (1991).

[7] D. H. Whittum, W. M. Sharp, S. S. Yu, M. Lampe, and G. Joyce, Phys. Rev. Lett. 67, 991 (1991).

[8] M. Lampe, G. Joyce, S. P. Slinker, and D. H. Whittum, Phys. Fluids B 5, 1888 (1993).

[9] A. A. Geraci and D. H. Whittum, Phys. Plasmas 7, 3431 (2000).

[10] S. Deng, C. D. Barnes, C. E. Clayton, C. O'Connell, F. J. Decker, R. A. Fonseca, C. Huang, M. J. Hogan, R. Iverson, D. K. Johnson, C. Joshi, T. Katsouleas, P. Krejcik, W. Lu, W. B. Mori, P. Muggli, E. Oz, F. Tsung, D. Walz, and M. Zhou, Phys. Rev. Lett. 96, 045001 (2006).

[11] C. Huang, W. Lu, M. Zhou, C. E. Clayton, C. Joshi, W. B. Mori, P. Muggli, S. Deng, E. Oz, T. Katsouleas, M. J. Hogan, I. Blumenfeld, F. J. Decker, R. Ischebeck, R. H. Iverson, N. A. Kirby, and D. Walz, Phys. Rev. Lett. 99, 255001 (2007).

[12] R. A. Fonseca, L. O. Silva, F. S. Tsung, V. K. Decyk, W. Lu, C. Ren, W. B. Mori, S. Deng, S. Lee, T. Katsouleas, and J.C. Adam, in Computational Science-ICCS 2002, Lecture Notes in Computer Science Vol. 2331, edited by P. Sloot, A. Hoekstra, C. Tan, and J. Dongarra (Springer,
Berlin, 2002), pp. 342-351; R. A. Fonseca, S. F. Martins, L. O. Silva, J. W. Tonge, F. S. Tsung, and W. B. Mori, Plasma Phys. Controlled Fusion 50, 124034 (2008); R. A. Fonseca, J. Vieira, F. Fiuza, A. Davidson, F. S. Tsung, W. B. Mori, and L. O. Silva, Plasma Phys. Controlled Fusion 55, 124011 (2013).

[13] T. J. Mehrling, R. A. Fonseca, A. Martinez de la Ossa, and J. Vieira (to be published).

[14] Y. Glinec, J. Faure, A. Lifschitz, J. M. Vieira, R. A. Fonseca, L. O. Silva, and V. Malka, Europhys. Lett. 81, 64001 (2008).

[15] J. Vieira, C.-K. Huang, W. B. Mori, and L. O. Silva, Phys. Rev. ST Accel. Beams 14, 071303 (2011).

[16] J. Vieira, W. B. Mori, and P. Muggli, Phys. Rev. Lett. 112, 205001 (2014).

[17] K. V. Lotov, Phys. Rev. E 69, 046405 (2004).

[18] A. Martinez de la Ossa, T. J. Mehrling, L. Schaper, M. J. V. Streeter, and J. Osterhoff, Phys. Plasmas 22, 093107 (2015).

[19] M. J. Hogan, T. O. Raubenheimer, A. Seryi, P. Muggli, T. Katsouleas, C. Huang, W. Lu, W. An, K. A. Marsh, W. B. Mori, C. E. Clayton, and C. Joshi, New J. Phys. 12, 055030 (2010).

[20] E. Adli, J.-P. Delahaye, S. J. Gessner, M. J. Hogan, T. Raubenheimer, W. An, C. Joshi, and W. Mori, arXiv: 1308.1145.

[21] V. E. Balakin, A. V. Novokhatsky, and V. P. Smirnov, eConf. Proc. C830811, 119 (1983).

[22] See Supplemental Material at http://link.aps.org/ supplemental/10.1103/PhysRevLett.118.174801 for further information regarding the physical and numerical setup, including Refs. [23-26].

[23] W. Lu, C. Huang, M. Zhou, W. B. Mori, and T. Katsouleas, Phys. Rev. Lett. 96, 165002 (2006).

[24] W. Lu, C. Huang, M. Zhou, M. Tzoufras, F. S. Tsung, W. B. Mori, and T. Katsouleas, Phys. Plasmas 13, 056709 (2006).

[25] S. A. Yi, V. Khudik, C. Siemon, and G. Shvets, Phys. Plasmas 20, 013108 (2013).

[26] R. Lehe, A. Lifschitz, C. Thaury, V. Malka, and X. Davoine, Phys. Rev. ST Accel. Beams 16, 021301 (2013).

[27] K. Floettmann, Phys. Rev. ST Accel. Beams 17, 054402 (2014).

[28] X. L. Xu, J. F. Hua, Y. P. Wu, C. J. Zhang, F. Li, Y. Wan, C.-H. Pai, W. Lu, W. An, P. Yu, M. J. Hogan, C. Joshi, and W. B. Mori, Phys. Rev. Lett. 116, 124801 (2016).

[29] L. Schaper, L. Goldberg, T. Kleinwächter, J.-P. Schwinkendorf, and J. Osterhoff, Nucl. Instrum. Methods Phys. Res., Sect. A 740, 208 (2014). 